\begin{tabular}{|c|c|c|c|c|c|c|}
\hline \multirow{4}{*}{ Impact Factor: } & ISRA (India) & $=3.117$ & SIS (USA) & $=0.912$ & ICV (Poland) & $=6.630$ \\
\hline & ISI (Dubai, UAE & $=0.829$ & РИНЦ (Russia & $=0.156$ & PIF (India) & $=1.940$ \\
\hline & GIF (Australia) & $=0.564$ & ESJI (KZ) & $=8.716$ & IBI (India) & $=4.260$ \\
\hline & JIF & $=1.500$ & SJIF (Morocco & $=\mathbf{5 . 6 6 7}$ & OAJI (USA) & $=0.350$ \\
\hline
\end{tabular}

\begin{tabular}{|c|c|}
\hline \multicolumn{2}{|c|}{$\begin{array}{l}\text { SOI: } 1.1 / \mathrm{TAS} \\
\text { International Scientific Journal } \\
\text { Theoretical \& Applied Science }\end{array}$} \\
\hline p-ISSN: 2308-4944 (print) & e-ISSN: 2409-0085 (online) \\
\hline Year: 2019 & Volume: 72 \\
\hline Published: 09.04 .2019 & http://T-Science.org \\
\hline
\end{tabular}

УДК 616.12
QR - Issue

QR - Article

\title{
RISK FACTORS OF DEVELOPMENT OF THE CARDIOVASCULAR DISEASES AND DIABETES MELLITUS TYPE 2 ON SCALES SCORE, FRAMINGHAM AND PROCAM SCALE
}

\begin{abstract}
This article discusses the literature review of risk factors, the development of cardiovascular diseases and type 2 diabetes mellitus on the SCORE, Framingham and PROCAM scales according to the PUBMED database. Many studies conducted by scientists of the world show the effectiveness of the results of the risk scales for the development of cardiovascular diseases and diabetes mellitus type 2 PROCAM, Framingham and SCORE in different countries varying.

Key words: SCORE, Framingham, PROCAM.

Language: Russian

Citation: Sharabitdinova, G. G. (2019). Risk factors of development of the cardiovascular diseases and diabetes mellitus type 2 on scales SCORE, FRAMINGHAM and PROCAM scale. ISJ Theoretical \& Applied Science, 04 (72), 30-33.

Soi: http://s-0-i.org/1.1/TAS-04-72-5 Doi: crossef https://dx.doi.org/10.15863/TAS.2019.04.72.5

\section{ФАКТОРЫ РИСКА РАЗВИТИЯ СЕРДЕЧНО СОСУДИСТЫХ \\ ЗАБОЛЕВАНИЙ И САХАРНОГО ДИАБЕТА 2 ТИПА \\ ПО ШКАЛАМ SCORE, FRAMINGHAM И РROCAM}

Аннотация: В данной статье рассматривается литературный обзор факторов риска, развитии сердечно-сосудистых заболеваний и сахарного диабета 2 типа по шкалам SCORE, Framingham и PROCAM по данным базы PUBMED. Множество исследований проведенное ученными мира, показывают эффективность результатов шкал риска развития сердечно-сосудистых заболеваний и сахарного диабета 2 muna PROCAM, Framingham и SCORE в разных странах неодинаковая.

Ключевые слова: SCORE, Framingham, PROCAM.
\end{abstract}

Introduction

Основными проблемами современной медицины остаются сердечно-сосудистые заболевания (СС3), сахарный диабет (СД) (в частности сахарный диабет 2 типа) и в последние годы уделяется большое внимание также метаболическому синдрому, связанному с абдоминальным ожирением [1-5].

По прогнозам ВОЗ к 2030 году от СС3 умрет 23.3млн. человек [6], а число больных сахарным диабетом возрастет на 55.2млн. человек [7]. По данным ВО3 в Европейских странах в отличие от стран СНГ (страны независимых государств) отмечается низкая доля смертности от ССЗ и СД. Низкий показатель смертности от СС3 отмечаются в странах Финляндия (44,5 на 100 тыс. населения), Германия (35,7 на 100 тыс. населения), Нидерландия (26,0 на 100 тыс. населения) В то время в таких странах как Литва, Латвия и Румыния этот показатель был намного выше (108,9-140,2 на 100 тыс. населения). Высокий показатель смертности от ССЗ и СД отмечается в России, Республике Беларусь, Украине и Республике Казахстан, (204,8-240,6 на 100 тыс. населения) [8].

В результате множество исследований показано, что восемь факторов риска: артериальная гипертензия, курение, гиподинамия, дислипидемия, гипергликемия, избыточная масса тела и нерациональное питание до 75\% обусловливают смертности от СС3 и СД 2 типа [9]. В течение многих лет факторы риска были 


\begin{tabular}{|c|c|c|c|c|c|c|}
\hline \multirow{4}{*}{ Impact Factor: } & ISRA (India) & $=3.117$ & SIS (USA) & $=0.912$ & ICV (Poland) & $=6.630$ \\
\hline & ISI (Dubai, UAE & $=0.829$ & РИНЦ (Russia) & $=0.156$ & PIF (India) & $=1.940$ \\
\hline & GIF (Australia) & $=0.564$ & ESJI (KZ) & $=8.716$ & IBI (India) & $=4.260$ \\
\hline & JIF & $=1.500$ & SJIF (Morocco & $=5.667$ & OAJI (USA) & $=0.350$ \\
\hline
\end{tabular}

выявлены во многих эпидемиологических исследованиях [10-14].

В клинической практике у пациентов, как правило, имеется сочетание двух и более факторов риска. В конце XX века разработаны многомерные модели оценки суммарного сердечно-сосудистого риска с учетом совокупности основных факторов, определяющих прогноз.

\section{Materials and Methods}

Европейская модель SCORE (Systematic Coronary Risk Evaluation) разработана экспертами Европейского общества кардиологов на базе данных 12 европейских когортных исследований с участием более 205 тыс. человек, в том числе 3325 человек из России [15]. Исследование началось с конца 70-х годах XX века и продолжалось 27 лет. Эта модель разработана на основе исследования европейской популяции и для нее. Поэтому имеет ряд отличий от американской модели Framingham. Так как шкала Framingham оценивает 10 летний риск развития фатальных и нефатальных сердечно-сосудистых осложнений, а модель SCORE определяет только 10 летний риск развития CC3.

Шкала Framingham - модель оценки суммарного риска развития СС3 и их осложнений. Она разработана на основе самого продолжительного эпидемиологического исследования (Framingham Heart Study, 19481984 гг.), которая началась в американском городе Фрамингем [16]. В исследовании участвовали 5209 человек от 30 до 62 лет. Благодаря этому исследованию были выявлены главные факторы риска сердечно-сосудистых заболеваний и общей сердечно-сосудистой смертности. Раньше шкалу Framingham применяли только для жителей Америки, где совпадали прогнозируемые и наблюдаемые результаты риска. Русифицированный вариант этой модели был разработан для России, но, в практической медицине широкого применения она не нашла. В дальнейшем европейскими экспертами были опубликованы работы, в которых анализировалась целесообразность применения шкалы Framingham в европейской популяции. Так, например, в British Regional Heart Study ее использование привело к завышению абсолютного риска коронарной смерти на $47 \%$, а суммарного показателя фатальных и нефатальных коронарных событий на $57 \%$.

Компьютерная программа PROCAM. Значительно более точные данные по определению суммарного риска дает математическая модель PROCAM в виде компьютерной программы CERCA (Coronary Events Risk Calculator) [17]. Она разработана на основе результатов проспективного исследования PROCAM - Prospective Cardiovascular Munster
Study, которая началась в городе Мюнстер (Германия) в 1979 г. В исследовании участвовали 21306 человек в возрасте от 40 до 65 лет. Эта шкала позволяет выявить факторы риска ишемической болезни сердца.

Целью исследования ученных Кательницкой и др. было оценить суммарный сердечнососудистый риск по шкале SCORE, до лечения и после лечения препаратом «Энам». В исследовании участвовали 143 больных с артериальной гипертензией. Результат исследования показал, что снижение артериального давления с помощью 4-недельной терапии препаратом «Энам» позволяет снизить суммарный риск СС3 на $21 \%$ от исходного положения [18].

В Бразилии ученные изучали риск развития CC3 по шкалам Framingham, PROCAM у ВИЧ инфицированных пациентов. В исследовании участвовали лица старше 19 лет (средний возраст $36,8 \pm 10,3), 76,9 \%$ пациентов составляли мужчины, $66,3 \%$ - получали антиретровирусную терапию, $47,8 \%$ пациентов имели абдоминальное ожирение, $23,1 \%$ были курильщиками, 20,0\% страдали артериальной гипертензией и у 2,0\% пациентов был сахарный диабет. У многих пациентов была дислипидемия (ХС ЛПНП >3,0 ммоль/л, Хс ЛПВП $<1,0$ ммоль/л). При оценке риска развития СС3 по шкале PROCAM показал, что в группе высоко риска доля пациентов самый низкий, по шкале Framingham наоборот, доля пациентов в группе высокого риска - высокий. Таким образом, шкала Framingham предсказывает более высокий риск развития ССЗ среди ВИЧ инфицированных [19].

В Турции Yalcin M. и другие провели исследования, целью которого является сравнение часто используемых оценочных шкал риска развития СС3. 350 пациентов, перенесших коронарную ангиографию, участвовали в исследовании. Риск развития СС3 оценивали с помощью шкал SCORE, Framingham и PROCAM. Оценка риска развития атеросклероза коронарных артерии могут предсказать все три модели. Однако шкала Framingham имеет лучшую прогностическую ценность, чем другие модели [20].

Аналогичное исследование провели в Нидерландах. Ученные сравнивали три шкалы риска развития CC3 SCORE, Framingham и PROCAM. В исследовании участвовали 1296 пациентов, перенесших коронарную ангиографию. Результат исследования показал, что модель Framingham является наиболее эффективной шкалой риска развития СС3 [21].

\section{Conclusion}

В Швейцарии риск развития СС3 у пациентов с атеросклерозом оценивали по четырем шкалам PROCAM, Framingham, SCORE и SMART. Изучая 


\begin{tabular}{|c|c|c|c|c|c|c|}
\hline \multirow{4}{*}{ Impact Factor: } & ISRA (India) & $=3.117$ & SIS (USA) & $=0.912$ & ICV (Poland) & $=6.630$ \\
\hline & ISI (Dubai, UAE & $=0.829$ & РИНЦ (Russia & $=0.156$ & PIF (India) & $=1.940$ \\
\hline & GIF (Australia) & $=0.564$ & ESJI (KZ) & $=8.716$ & IBI (India) & $=4.260$ \\
\hline & JIF & $=1.500$ & SJIF (Morocco & $=\mathbf{5 . 6 6 7}$ & OAJI (USA) & $=0.350$ \\
\hline
\end{tabular}

средние показатели всех четырех шкал, ученные в своем исследовании показали, что шкала SMART является лучшей моделью для оценки риска развития СС3 [22].

Английские ученные применили шкалы риска развития СС3 у 234 пациентов и в результатах своего исследования показали, что шкала Framingham является наиболее эффективной, чем шкала PROCAM [23].

Таким образом, эффективность шкал риска развития CC3 PROCAM, Framingham и SCORE в разных странах неодинаковая.

\section{References:}

1. Williams, B. (2009). The changing face of hypertension treatment: treatment strategies from the $2007 \mathrm{ESH} / \mathrm{ESC}$ hypertension Guidelines. J. Hypertens, №27, 19-26.

2. Lazzini, A., \& Lazzini, S. (2009). Cardiovascular disease: an economical perspective. Curr. Pharm. Des. №10, 11421156.

3. Bokeriya, L. A., et al. (n.d.). «Serdechno sosudistyye zabolevaniya na rubezhe vekov: smertnost', raspostranennost', faktory riska».

4. Shinbolatova, A., Kulzhanov, M., Aringazina, A., \& Nurbakhyt, A. (2014). Screening of Arterial Hypertension in the Republic of Kazakhstan: Advantages, Disadvantages and Ways of Improving. Iran J Public Health. №43(12), 1695-701.

5. (2013). Health of population and health care in the Republic of Kazakhstan in 2013. Official Statistic MedInform. (p.258). Astana. Available on www.medinfo.kz

6. (2013). Kardiologiya, Materialy VOZ Informatsionnyy byulleten' №317, Mart 2013.

7. Cho, N. H. (2013), Executive summary International Diabetes Federations IDF Diabetes Atlas. № 6, 11 .

8. Kalashnikov, K. N., Lastochkina, N. A., \& Duganov, M. D. (2015). Osobennosti zabolevayemosti i smertnosti v Volgogradskoy oblasti ot serdechno-sosudistykh zabolevaniy. Gumanitarnyye nauchnyye issledovaniya. №1, 16-23.

9. Boytsov, S. A., \& Chuchalin, A. G. (2013). Profilaktika khronicheskikh neinfektsionnykh zabolevaniy. Monografiya. (p.7).

10. Li, Y., et al. (2015). Birth weight and later life adherence to unhealthy lifestyles in predicting type 2 diabetes: prospective cohort study. $B M J$, №21, 351 .

11. Mons, U., et al. (2015). Impact of smoking and smoking cessation on cardiovascular events and mortality among older adults: meta-analysis of individual participant data from prospective cohort studies of the CHANCES consortium. BMJ, №4, 350 .

12. Kumar, R., \& Indian, J. (2015). Anthropometric and behavioral risk factor for non-communicable diseases: a cluster survey from rural Wardha. Public Health. №59(1), 61-64.

13. Anghel, L., Ghiuru, R., \& Gavrilescu, C. M. (2010). Dislypidemia as a risk factor for cardiovascular disease. Rev Med Chir Soc Med Nat Iasi. №114(4), 977-981.

14. Subramoney, S., Bjorkelund, C., Guo, X., Skoog, I., Bosaeus, I., \& Lissner, L. (2014). Agerelated differences in recommended anthropometric cut-off point validity to identify cardiovascular risk factors in ostensibly healthy women. Scand J Public Health. №42(8), 827833.

15. Conroy, R. M., et al. (2003). Estimation of tenyear risk of fatal cardiovascular disease in Europa: the SCORE project. Eur Heart J, №24, 987-1003.

16. Anderson, K. M., Odel, P. M., Wilson, P. W., \& Kannel, W. B. (1991). Cardiovascular disease risk profiles. Am Heart J, №121, 293-298.

17. Assmann, G., Cullen, P., \& Schulte, H. (1998). The Munster Heart Study (PROCAM). European Heart J, №19, 2-11.

18. Katel'nitskaya, L. I., Khaisheva, L. A., \& Glova, S. Y. (2007). Summarnyy serdechno-sosudistyy risk u bol'nykh gipertonicheskoy bolezn'yu: vozmozhnosti ingibitorov APF. Ratsional'naya Farmakoterapiya $v$ kardiologii №5, tom 3, 9-14.

19. Nery, M. W., et al. (2013). Cardiovascular risk assessment: a comparison of the Framingham, PROCAM, and DAD equations in HIV-infected persons. ScientificWorldJournal. №21, 21282136.

20. Yalcin, M., et al. (2012). Cardiovascular risk scores for coronary atherosclerosis. J. Acta Cardiol. №67(5), 557-563.

21. Versteylen, M.O., Joosen, I.A., Shaw, L.J., Narula, J., \& Hofstra, L. (2011). Comparison of Framingham, PROCAM, SCORE, and Diamond 


\begin{tabular}{|c|c|c|c|c|c|c|}
\hline \multirow{4}{*}{ Impact Factor: } & ISRA (India) & $=3.117$ & SIS (USA) & $=0.912$ & ICV (Poland) & $=6.630$ \\
\hline & ISI (Dubai, UAE & $=0.829$ & РИНЦ (Russia & $=0.156$ & PIF (India) & $=1.940$ \\
\hline & GIF (Australia) & $=0.564$ & ESJI (KZ) & $=8.716$ & IBI (India) & $=4.260$ \\
\hline & JIF & $=1.500$ & SJIF (Morocco & $=5.667$ & OAJI (USA) & $=0.350$ \\
\hline
\end{tabular}

Forrester to predict coronary atherosclerosis and cardiovascular events. J Nucl Cardiol. №18(5), 904-911.

22. Uthoff, H., et al. (2010) PROCAM-, Framingham-, SCORE- and SMART-risk score for predicting cardiovascular morbidity and mortality in patients with overt atherosclerosis. Vasa. №39(4), 325-333.
23. Vrentzos, G.E., et al. (2007). Predicting coronary heart disease risk using the Framingham and PROCAM equations in dyslipidaemic patients without overt vascular disease. Int J Clin Pract. №61(10), 1643-1653. 